\title{
Anti-de Sitter/ boundary conformal field theory correspondence in the non-relativistic limit
}

\author{
M. R. Setare 1 \\ V. Kamali \\ Department of Science, Payame Noor University, Bijar, Iran
}

\begin{abstract}
Boundary conformal field theory (BCFT) is the study of conformal field theory (CFT) in semi-infinite space-time. In non-relativistic limit ( $x \rightarrow \epsilon x, t \rightarrow t, \epsilon \rightarrow 0$ ), boundary conformal algebra changes to boundary Galilean conformal algebra (BGCA). In this work, some aspects of AdS/BCFT in non-relatvistic limit were explored. We constrain correlation functions of Galilean conformal invariant fields with BGCA generators. For a situation with a boundary condition at surface $x=0(z=\bar{z})$, our result is agree with non-relativistic limit of BCFT two-point function. We also, introduce holographic dual of boundary Galilean conformal field theory.
\end{abstract}

\footnotetext{
${ }^{1}$ rezakord@ipm.ir

${ }^{2}$ vkamali1362@gmail.com
} 


\section{Introduction}

Recently, there has been some interest in extending the AdS/CFT correspondence to non-relativistic field theories [1, 2], where the non-relativistic conformal symmetry was obtained by a parametric contraction of the relativistic conformal group. Galilean conformal algebra (GCA) arises as a contraction relativistic conformal algebras [1, 3, 4], where in $d=4$ the Galilean conformal group is a fifteen parameter group which contains the ten parameter Galilean subgroup. Beside Galilean conformal algebra, there is another non-relativistic algebra, the twelve parameter Schrödinger algebra [5, 6. The dilatation generator in the Schrödinger group scales space and time differently, $x_{i} \rightarrow \lambda x_{i}, t \rightarrow \lambda^{2} t$, but in contrast the corresponding generator in GCA scales space and time in the same way, $x_{i} \rightarrow \lambda x_{i}, t \rightarrow \lambda t$. Infinite dimensional Galilean conformal group has been reported in [3]. The generators of this group are : $L^{n}=-(n+1) t^{n} x_{i} \partial_{i}-t^{n+1} \partial_{t}, M_{i}^{n}=t^{n+1} \partial_{i}$ and $J_{i j}^{n}=-t^{n}\left(x_{i} \partial_{j}-x_{j} \partial_{i}\right)$ for an arbitrary integer $n$, where $i$ and $j$ are specified by the spatial directions which obey commutation relation of the Virasoro-Kac-Moody algebra [3]. There is a finite dimensional subgroup of the infinite dimensional Galilean conformal group which is generated by $\left(J_{i j}^{0}, L^{ \pm 1}, L^{0}, M_{i}^{ \pm 1}, M_{i}^{0}\right)$. These generators are obtained by contraction ( $t \rightarrow t, x_{i} \rightarrow \epsilon x_{i}, \epsilon \rightarrow 0, v_{i} \sim \epsilon$ ) of the relativistic conformal generators. The gravity dual of finite GCA was considered in [3, 4, 7, and the metric with finite 2d GCA isometry was obtained in [8].

The presence of free surfaces or walls in macroscopic systems which are at the critical point, lead to the large variety of physical effects. Since, using boundary condition effect is shown to be very helpful in various branch in physics, the systems with boundary conditions have been considered by both theorists [9] and experimentalists [10]. The situation with walls or free surfaces opens a new area in condensed matter physics [11]. In reference [12, the research on semi-infinite systems which exhibits a non-equilibrium bulk phase transitions was initiated and the effects of boundary condition on direct percolation were considered.

Holographic dual of a conformal field theory with a boundary (BCFT) was proposed in [13. The main idea of AdS/BCFT correspondence was started with asymptotically AdS geometry with Neumann boundary condition on the metric as one approaches to the boundary [13, 14]. The geometry is modified by imposing two different boundary conditions on the metric. The boundary is divided into two parts $\partial M=N \bigcup Q$ where $\partial Q=\partial N$ [13. The metric has Neumann boundary condition on $Q$ and Dirichlet boundary condition on $N$. With this boundary condition the AdS geometry is divided 
into two parts and the gravitational theory lives in one part of this space. This modified geometry could provide a holographic dual for BCFT. Boundary conformal field theory (BCFT) is defined in domains with a boundary [15. In this work we extend AdS/BCFT correspondence to non-relativistic version. When non-relativistic CFT lives in semi-infinite space, one sector of Galilean conformal group is removed. For example, if we have a boundary condition on surface $z=\bar{z}(t-x=t+x)$ or $x=0$, translation, boost and spatial-special conformal transformations are removed. So, two-point function in this situation is completely different from situation without boundary condition (free space). Two-point function of BCFT in the situation with a boundary condition at surface $z=\bar{z}$ was calculated in [16, 17]. In this paper we calculate two-point and three point functions of BGCA from gravity dual [7] and quantum field theory method in the boundary [18, 19]. Our results agree with results [16, 17] in non-relativistic limit. We also, introduce holographic dual of non-relativistic limit of BCFT (BGCA). The paper organized as follow: In section 2 we give a brief review of $2 \mathrm{~d}$ GCA. In section 3 we calculate two-point and three point correlation functions of Galilean conformal invariant fields in semi-infinite space. In section 4 we introduce holographic dual of non-relativistic BCFT, then we calculate two-point function from gravity dual. Finally, in section 5, we close by some concluding remarks.

\section{GCA in $2 d$}

Galilean conformal algebra (GCA) in $2 \mathrm{~d}$ is obtained by contracting $2 \mathrm{~d}$ conformal symmetry [18]. Two-dimensional Conformal algebra is described by two copies of Virasoro algebra. In quantum field theory (QFT) level, twodimensional $(z=t+x, \bar{z}=t-x)$ CFT generators

$$
\mathcal{L}_{n}=z^{n+1} \partial_{z}, \quad \overline{\mathcal{L}}_{n}=\bar{z}^{n+1} \partial_{\bar{z}},
$$

obey the Virasoro algebra

$$
\begin{aligned}
& {\left[\mathcal{L}_{m}, \mathcal{L}_{n}\right]=(n-m) \mathcal{L}_{m+n}+\frac{c_{R}}{12} m\left(1-m^{2}\right) \delta_{m+n, 0}} \\
& {\left[\overline{\mathcal{L}}_{m}, \overline{\mathcal{L}}_{n}\right]=(n-m) \overline{\mathcal{L}}_{m+n}+\frac{c_{L}}{12} m\left(1-m^{2}\right) \delta_{m+n, 0}}
\end{aligned}
$$

In non-relativistic limit $(t \rightarrow t, x \rightarrow \epsilon x$ with $\epsilon \rightarrow 0)$, the GCA generators $L_{n}$ and $M_{n}$ are constructed by Virasoro generators

$$
\begin{aligned}
& L_{n}=\lim _{\epsilon \rightarrow 0}\left(\mathcal{L}_{n}+\overline{\mathcal{L}}_{n}\right)=(n+1) t^{n} \partial_{x}+t^{n+1} \partial_{x}, \\
& M_{n}=-\lim _{\epsilon \rightarrow 0} \epsilon\left(\mathcal{L}_{n}-\overline{\mathcal{L}}_{n}\right)=-t^{n+1} \partial_{x} .
\end{aligned}
$$


From Eqs.(2) and (3), one obtains centrally extended 2d GCA

$$
\begin{aligned}
& {\left[L_{m}, L_{n}\right]=(n-m) L_{m+n}+C_{1} m\left(1-m^{2}\right) \delta_{m+n, 0},} \\
& {\left[L_{m}, M_{n}\right]=(n-m) M_{m+n}+C_{2} m\left(1-m^{2}\right) \delta_{m+n, 0},} \\
& {\left[M_{n}, M_{m}\right]=0 .}
\end{aligned}
$$

The GCA central charges $\left(C_{1}, C_{2}\right)$ are related to CFT central charges $\left(c_{L}\right.$, $\left.c_{R}\right)$ as:

$$
C_{1}=\lim _{\epsilon \rightarrow 0} \frac{c_{L}+c_{R}}{12}, \quad C_{2}=\lim _{\epsilon \rightarrow 0}\left(\epsilon \frac{c_{L}-c_{R}}{12}\right) .
$$

From above equations, for a non-zero and finite $\left(C_{2}, C_{1}\right)$ in the limit $\epsilon \rightarrow 0$, it can be seen that we need $c_{L}-c_{R} \propto O\left(\frac{1}{\epsilon}\right)$ and $c_{L}+c_{R} \propto O(1)$. Similarly, rapidity $\xi$ and scaling dimensions $\Delta$, which are the eigenvalues of $M_{0}$ and $L_{0}$ respectively, are given by

$$
\Delta=\lim _{\epsilon \rightarrow 0}(h+\bar{h}), \quad \xi=-\lim _{\epsilon \rightarrow 0} \epsilon(h-\bar{h}),
$$

where $h$ and $\bar{h}$ are eigenvalues of $\mathcal{L}_{0}$ and $\overline{\mathcal{L}}_{0}$ respectively. Equation (6) tells us that, $h+\bar{h}$ is of order $O(1)$ while $h-\bar{h}$ must be order $O\left(\frac{1}{\epsilon}\right)$, for the finite $\Delta, \xi$.

\section{Correlation functions in semi-infinite space}

In this section we find the correlation functions in semi-infinite space with a boundary condition at surface $z=\bar{z}$. We now turn to derive the consequences of Galilean conformal invariance for the correlation. In general, we expect a quasi-primary field $\mathcal{O}$ to be characterized by its Galilean conformal dimension $\Delta$ and rapidity $\xi$ (these fields are invariant under finite sub-group that is generated by sub-algebra $\left.\left\{L_{-1}, M_{-1}, L_{0}, M_{0}, L_{1}, M_{1}\right\}\right)$. We would like to find the form of two-point and three-point functions of the Galilean conformal invariant operators in semi-infinite space. Firstly, we find the form of the commutators $\left[\mathcal{L}_{n}, \mathcal{O}\right]$ and $\left[\overline{\mathcal{L}}_{n}, \mathcal{O}\right]$, then we obtain the form of $\left[L_{n}, \mathcal{O}\right]$ and $\left[M_{n}, \mathcal{O}\right]$ as following

$$
\begin{aligned}
& {\left[\mathcal{L}_{n}, \mathcal{O}(z, \bar{z})\right]=\left[\mathcal{L}_{n}, U \mathcal{O}(0) U^{-1}\right]=\left[\mathcal{L}_{n}, U\right] \mathcal{O}(0) U^{-1}+U \mathcal{O}(0)\left[\mathcal{L}_{n}, U^{-1}\right]} \\
& +U\left[\mathcal{L}_{n}, \mathcal{O}(0)\right] U^{-1}=U\left\{U^{-1} \mathcal{L}_{n} U-\mathcal{L}_{n}\right\} \mathcal{O}(0) U^{-1} \\
& \quad+U \mathcal{O}(0)\left\{\mathcal{L}_{n}-U^{-1} \mathcal{L}_{n} U\right\} U^{-1}+\delta_{n, 0} h \mathcal{O}(z, \bar{z})
\end{aligned}
$$


$U$ and $\mathcal{O}(z, \bar{z})$ are defined as

$$
\mathcal{O}(z, \bar{z})=U \mathcal{O}(0) U^{-1} \quad \text { where } \quad U=e^{z \mathcal{L}_{-1}+\bar{z} \overline{\mathcal{L}}_{-1}}
$$

By using the Hausdorff formula we get

$$
\begin{aligned}
U^{-1} \mathcal{L}_{n} U & =e^{-z \mathcal{L}_{-1}-\bar{z} \overline{\mathcal{L}}_{-1}} \mathcal{L}_{n} e^{z \mathcal{L}_{-1}+\bar{z} \overline{\mathcal{L}}_{-1}}=e^{-z \mathcal{L}_{-1}} \mathcal{L}_{n} e^{z \mathcal{L}_{-1}} \\
& =\mathcal{L}_{n}+\left[\mathcal{L}_{n}, z \mathcal{L}_{-1}\right]+\frac{1}{2 !}\left[\left[\mathcal{L}_{n}, z \mathcal{L}_{-1}\right], z \mathcal{L}_{-1}\right]+\ldots \\
& =\sum_{k=0}^{n+1} \frac{(n+1) !}{(n+1-k) ! k !}(z)^{k} \mathcal{L}_{n-k}
\end{aligned}
$$

and

$$
\mathcal{L}_{n}^{\prime}=U^{-1} \mathcal{L}_{n} U-\mathcal{L}_{n}=\sum_{k=1}^{n+1} \frac{(n+1) !}{(n+1-k) ! k !}(z)^{k} \mathcal{L}_{n-k}
$$

The Eq.(17) gives us

$$
\begin{aligned}
& {\left[\mathcal{L}_{n}, \mathcal{O}(z, \bar{z})\right]=U\left\{\left[\mathcal{L}_{n}^{\prime}, \mathcal{O}(0)\right]+\delta_{n, 0} h \mathcal{O}(0)\right\} U^{-1}} \\
& =z^{n+1}\left[\mathcal{L}_{-1}, \mathcal{O}(z, \bar{z})\right]+z^{n}(n+1) U\left[\mathcal{L}_{0}, \mathcal{O}(0)\right] U^{-1}
\end{aligned}
$$

Now we have $\left[\mathcal{L}_{-1}, \mathcal{O}\right]=\partial_{z} \mathcal{O}$ and $\left[\mathcal{L}_{0}, \mathcal{O}\right]=h \mathcal{O}\left(\mathcal{L}_{0}\right.$ and $\mathcal{L}_{-1}$ generate $z$-dilatation and $z$-translation, respectively). Hence we obtain (for $n \geq-1$ )

$$
\left[\mathcal{L}_{n}, \mathcal{O}(z, \bar{z})\right]=\left(z^{n+1} \partial_{z}+(n+1) h z^{n}\right) \mathcal{O}
$$

We can exchange $\mathcal{L}_{n}$ with $\overline{\mathcal{L}}_{n}$ and using the above steps (7)-(11). We get

$$
\left[\overline{\mathcal{L}}_{n}, \mathcal{O}(z, \bar{z})\right]=\left(\bar{z}^{n+1} \partial_{\bar{z}}+(n+1) h \bar{z}^{n}\right) \mathcal{O}
$$

From Eqs.(12), (13) and by using the definitions of $L_{n}$ and $M_{n}$ (3), we can find the form of commutators $\left[L_{n}, \mathcal{O}\right]$ and $\left[M_{n}, \mathcal{O}\right]$

$$
\begin{aligned}
& {\left[L_{n}, \mathcal{O}\right]=\left(t^{n+1} \partial_{t}+(n+1) t^{n} x \partial_{x}+(n+1)\left(t^{n} \Delta-n x t^{n-1} \xi\right)\right) \mathcal{O}} \\
& {\left[M_{n}, \mathcal{O}\right]=\left(t^{n+1} \partial_{x}-(n+1) t^{n} \xi\right) \mathcal{O}}
\end{aligned}
$$

Correlation functions of GCA are constrained by the above equations in free space [19]. If we have a boundary in $x$ direction, symmetries in this direction is removed obviously. So, in the situation with a boundary condition at 
surface $x=0(z=\bar{z})$, Galilean symmetry group reduces to one copy of nonrelativistic version of Virasoro group which is generated by $L_{n}$ [16, 17]. We can use this subgroup to calculate two-point function. Firstly, we consider the invariance under time translation which is generated by $L_{-1}$

$$
<0\left|\left[L_{-1}, G\right]\right| 0>=0 \Rightarrow G=G\left(x_{1}, x_{2}, \tau\right) \quad \tau=t_{1}-t_{2}
$$

where $G=<\mathcal{O}_{1} \mathcal{O}_{2}>$ is two-point function of two quasi-primary operators $\mathcal{O}_{1}$ and $\mathcal{O}_{2}$. Invariance under dilatation constrains two-point function as

$$
\begin{aligned}
& <0\left|\left[L_{0}, G\right]\right| 0>=0 \\
& \Rightarrow \sum_{i=1}^{2}\left(t_{i} \partial_{t_{i}}+x_{i} \partial_{x_{i}}+\Delta_{i}\right) G=0 \\
& \left(\tau \partial_{\tau}+x_{1} \partial_{x_{1}}+x_{2} \partial_{x_{i}}+\Delta\right) G=0 \quad \Delta=\Delta_{1}+\Delta_{2}
\end{aligned}
$$

Invariance under spatial component of special conformal transformation is

$$
\begin{aligned}
& \quad<0\left|\left[L_{1}, G\right]\right| 0>=0 \\
& \Rightarrow \sum_{i=1}^{2}\left(t_{i}^{2} \partial_{t_{i}}+2 t_{i} x_{i} \partial_{x_{i}}+2 t_{i} \Delta_{i}-x_{i} \xi_{i}\right) G \\
& \quad=\left(\left(t_{1}^{2}-t_{2}^{2}\right) \partial_{\tau}+2\left(t_{1} x_{1} \partial_{x_{1}}+t_{2} x_{2} \partial_{x_{2}}\right)\right. \\
& \left.\quad+2\left(t_{1} \Delta_{1}+t_{2} \Delta_{2}-x_{1} \xi_{1}-x_{2} \xi_{2}\right)\right) G \\
& =\left(\tau^{2} \partial_{\tau}+2 t_{2}\left(\tau \partial_{\tau}+x_{1} \partial_{x_{1}}+x_{2} \partial_{x_{2}}\right)\right. \\
& \left.\quad-2\left(x_{1} \xi_{1}+x_{2} \xi_{1}\right)+2 \tau x_{1} \partial_{x_{2}}+2\left(t_{1} \Delta_{1}+t_{2} \Delta_{2}\right)\right) G \\
& =\left(\tau^{2} \partial_{\tau}-2\left(x_{1} \xi_{1}+x_{2} \xi_{1}\right)+2 \tau x_{1} \partial_{x_{2}}+2 \tau \Delta_{1}\right) G=0
\end{aligned}
$$

where in the last step, Eq.(16) was used. We make the following ansatz

$$
G\left(x_{1}, x_{2}, \tau\right)=\tau^{-2 \Delta_{1}} G^{\prime}(u, v), \quad u=\frac{x_{1}}{\tau}, \quad v=\frac{x_{2}}{\tau}
$$

so, Eq. (17) gives

$$
\left(u \partial_{u}-v \partial_{v}-2\left(u \xi_{1}+v \xi_{2}\right)\right) G^{\prime}(u, v)=0,
$$

Solution of this equation is [9, 20]

$$
G^{\prime}(u, v)=\chi(u v) \exp \left(2\left(u \xi_{1}-v \xi_{2}\right)\right)
$$

where $\chi$ is an arbitrary function. The final result for two-point function is

$$
G\left(x_{1}, x_{2}, \tau\right)=\delta_{\Delta_{1}, \Delta_{2}} \tau^{-2 \Delta} \chi\left(\frac{x_{1} x_{2}}{\tau^{2}}\right) \exp \left(\frac{2}{\tau}\left(x_{1} \xi_{1}-x_{2} \xi_{2}\right)\right)
$$


where $\Delta=\Delta_{1}=\Delta_{2}$. It is clear that, two-point function near the boundary is different from other places [19]. Two-point function of BCFT for scalar fields was calculated in [16, 17]

$$
\begin{aligned}
G\left(z_{1}, z_{2}, \bar{z}_{1}, \bar{z}_{2}\right)= & \frac{1}{4}\left(\frac{1}{\left|z_{1}-z_{2}\right|^{2 \Delta}}+\frac{1}{\left|\bar{z}_{1}-\bar{z}_{2}\right|^{2 \Delta}}\right. \\
& \left.+\frac{1}{\left|z_{1}-\bar{z}_{2}\right|^{2 \Delta}}+\frac{1}{\left|\bar{z}_{1}-z_{2}\right|^{2 \Delta}}\right)
\end{aligned}
$$

In non-relativistic limit $(t \rightarrow t, x \rightarrow \epsilon x)$ we have

$$
\begin{aligned}
& \lim _{\epsilon \rightarrow 0}\left(z_{1}-z_{2}\right)=\lim _{\epsilon \rightarrow 0}\left(t_{1}+\epsilon x_{1}-t_{2}-\epsilon x_{2}\right)=t_{1}-t_{2} \\
& \lim _{\epsilon \rightarrow 0}\left(\bar{z}_{1}-\bar{z}_{2}\right)=\lim _{\epsilon \rightarrow 0}\left(t_{1}-\epsilon x_{1}-t_{2}+\epsilon x_{2}\right)=t_{1}-t_{2} \\
& \lim _{\epsilon \rightarrow 0}\left(z_{1}-\bar{z}_{2}\right)=\lim _{\epsilon \rightarrow 0}\left(t_{1}+\epsilon x_{1}-t_{2}+\epsilon x_{2}\right)=t_{1}-t_{2} \\
& \lim _{\epsilon \rightarrow 0}\left(\bar{z}_{1}-z_{2}\right)=\lim _{\epsilon \rightarrow 0}\left(t_{1}-\epsilon x_{1}-t_{2}-\epsilon x_{2}\right)=t_{1}-t_{2}
\end{aligned}
$$

From above equations we obtain

$$
\lim _{\epsilon \rightarrow 0} G\left(z_{1}, z_{2}, \bar{z}_{1}, \bar{z}_{2}\right)=\delta_{\Delta_{1}, \Delta_{2}} \tau^{-2 \Delta}
$$

which is agree with our result (21). (For scalar field $\xi_{i}$ is equal to zero.)

Now using above method, we calculate three-point correlation function in semi-infinite space-time with a boundary condition at surface $x=0$.

Consider the three-point function as

$$
G\left(x_{1}, x_{2}, x_{3}, t_{1}, t_{2}, t_{3}\right)=<\phi_{1}\left(x_{1}, t_{1}\right) \phi_{2}\left(x_{2}, t_{2}\right) \phi_{3}\left(x_{3}, t_{3}\right)>
$$

where $\phi_{1}, \phi_{2}$ and $\phi_{3}$ are Galilean conformal invariant fields. Invariance under the time translation symmetry implies $G=G\left(x_{1}, x_{2}, x_{3}, \tau, \sigma\right)$ where $\tau=$ $t_{1}-t_{3}$ and $\sigma=t_{2}-t_{3}$. We constrain $G$ by scale invariance as

$$
\begin{aligned}
& <0\left|\left[L_{0}, \phi_{1} \phi_{2} \phi_{3}\right]\right| 0>=0 \\
\Rightarrow & \sum_{i=1}^{i=3}\left(t_{i} \partial_{t_{i}}+x_{i} \partial_{i}+\Delta_{i}\right) G \\
= & \left(\tau \partial_{\tau}+\sigma \partial_{\sigma}+x_{1} \partial_{x_{1}}+r_{2} \partial_{x_{2}}+x_{3} \partial_{x_{3}}+\Delta_{1}+\Delta_{2}+\Delta_{3}\right) G=0
\end{aligned}
$$


From the invariance under the time component of non-relativistic special conformal transformation we get

$$
\begin{aligned}
& <0\left|\left[L_{1}, \phi_{1} \phi_{2} \phi_{3}\right]\right| 0>=0 \\
& \Rightarrow \sum_{i=1}^{i=3}\left(t_{i}^{2} \partial_{t_{i}}+2 t_{i} x_{i} \partial_{i}+2 t_{i} \Delta_{i}-x_{i} \xi_{i}\right) G \\
= & \left(\left(t_{1}^{2}-t_{3}^{2}\right) \partial_{\tau}+\left(t_{2}^{2}-t_{3}^{2}\right) \partial_{\sigma}+2\left(t_{1} x_{1} \partial_{x_{1}}+t_{2} x_{2} \partial_{x_{2}}+t_{3} x_{3} \partial_{3}\right)\right. \\
+ & \left.2\left(t_{1} \Delta_{1}+t_{2} \Delta_{2}+t_{3} \Delta_{3}\right)-x_{1} \xi_{1}-x_{2} \xi_{2}-x_{3} \xi_{3}\right) G \\
= & \left(\tau^{2} \partial_{\tau}+\sigma^{2} \partial_{\sigma}+2 t_{3}\left(\tau \partial_{\tau}+\sigma \partial_{\sigma}+x_{1} \partial_{x_{1}}+x_{2} \partial_{x_{2}}+x_{3} \partial_{3}\right)\right. \\
- & \left.x_{1} \xi_{1}-r_{2} \xi_{1}-x_{3} \xi_{3}+2 \tau x_{1} \partial_{x_{1}}+2 \sigma x_{2} \partial_{x_{2}}+2\left(t_{1} \Delta_{1}+t_{2} \Delta_{2}+t_{3} \Delta_{3}\right)\right) G \\
= & \left(\tau^{2} \partial_{\tau}-x_{1} \xi_{1}-x_{2} \xi_{2}-r_{3} \xi_{3}+2 \tau x_{1} \partial_{x_{1}}\right. \\
& \left.+\sigma^{2} \partial_{\sigma}+2 \sigma x_{2} \partial_{x_{2}}+2 \tau \Delta_{1}+2 \sigma \Delta_{2}\right) G=0
\end{aligned}
$$

where in the last equation we have used Eq.(26). We make the ansatz

$$
G=\delta_{\Delta_{1}+\Delta_{2}, \Delta_{3}} \tau^{-2 \Delta_{1}} \sigma^{-2 \Delta_{2}} G^{\prime}
$$

and simplify the above equations as

$$
\begin{aligned}
& \left(\tau^{2} \partial_{\tau}+2 \tau x_{1} \partial_{x_{1}}-x_{1} \xi_{1}-x_{3} \xi_{3}\right) G_{1}^{\prime}=0 \\
& \left(\tau \partial_{\tau}+x_{1} \partial_{x_{1}}+x_{3} \partial_{x_{3}}\right) G_{1}^{\prime}=0 \\
& \left(\sigma^{2}+2 \sigma x_{2} \partial_{x_{2}}-x_{2} \xi_{2}\right) G_{2}^{\prime}=0 \\
& \left(\sigma \partial_{\sigma}+x_{2} \partial_{x_{2}}\right) G_{2}^{\prime}=0
\end{aligned}
$$

where $G^{\prime}=G_{1}^{\prime}\left(x_{1}, x_{3}, \tau\right) G_{2}^{\prime}\left(x_{2}, \sigma\right)$, or

$$
\begin{aligned}
& \left(\sigma^{2} \partial_{\sigma}+2 \sigma x_{2} \partial_{x_{2}}-x_{2} \xi_{2}-x_{3} \xi_{3}\right) G_{1}^{\prime}=0 \\
& \left(\sigma \partial_{\sigma}+x_{2} \partial_{x_{2}}+x_{3} \partial_{x_{3}}\right) G_{1}^{\prime}=0 \\
& \left(\tau^{2}+2 \tau x_{1} \partial_{x_{1}}-x_{1} \xi_{1}\right) G_{2}^{\prime}=0 \\
& \left(\tau \partial_{\tau}+x_{1} \partial_{x_{1}}\right) G_{2}^{\prime}=0
\end{aligned}
$$

where $G^{\prime}=G_{1}^{\prime}\left(x_{2}, x_{3}, \sigma\right) G_{2}^{\prime}\left(x_{1}, \tau\right)$. By using the method of characteristic [20], we may found the general solution of these equations

$$
\begin{aligned}
& G= \delta_{\Delta_{1}+\Delta_{2}, \Delta_{3}}\left(t_{1}-t_{3}\right)^{-2 \Delta_{1}}\left(t_{2}-t_{3}\right)^{-2 \Delta_{2}} \exp \left(\frac{x_{1} \xi_{1}}{t_{1}-t_{3}}+\frac{x_{2} \xi_{2}}{t_{2}-t_{3}}\right) \\
&\left(\chi_{1}\left(\frac{x_{1} x_{3}}{\left(t_{1}-t_{3}\right)^{2}}\right) \exp \left(-\frac{x_{3} \xi_{3}}{t_{1}-t_{3}}\right)+\chi_{2}\left(\frac{x_{2} x_{3}}{\left(t_{2}-t_{3}\right)^{2}}\right) \exp \left(-\frac{x_{3} \xi_{3}}{t_{2}-t_{3}}\right)\right) \\
& \quad+\Sigma(\text { exchanging } \quad 2 \leftrightarrow 3 \text { or } 1 \leftrightarrow 3)
\end{aligned}
$$

where $\chi_{1}$ and $\chi_{2}$ are arbitrary functions. Three-point function near the boundary is obviously different from other places. 


\section{Holographic dual of non-relativistic BCFT}

Recently, holographic dual of BCFT was considered [13, 14, 16]. Ad $S_{3}$ with Neumann boundary condition at surface $z=\bar{z}$ is holographic dual of $B C F T_{2}$. In this situation the symmetry group of boundary conformal field theory is generated by one copy of Virasoro algebra [16. We introduce non-relativistic version of this gravity dual as a holographic dual of nonrelativistic BCFT. The $A d S_{3}$ metric in Poincare coordinates is

$$
d s^{2}=\frac{1}{r^{2}}\left(-d t^{2}+d r^{2}+d x^{2}\right)
$$

where $r$ is a radial coordinate and $(x, t)$ are boundary coordinate. In the Eddington-Finkelstein coordinates which define by $r=r^{\prime}$ and $t=t^{\prime}+r^{\prime}$ the $A d S_{3}$ metric is given by

$$
d s^{2}=\frac{1}{r^{2}}\left(d t^{2}-2 d t d r+d x^{2}\right)
$$

The Killing vectors of $A d S_{3}$ read as

$$
\begin{aligned}
& P=\partial_{x} \quad B=(t-r) \partial_{x}-x \partial_{t} \\
& K_{x}=\left(t^{2}-2 t r-x^{2}\right) \partial_{x}+2 t x \partial_{t}+2 r x \partial_{r}+2 x^{2} \partial_{x} \\
& H=-\partial_{t} \quad D=-t \partial_{t}-r \partial_{r}-x \partial_{x} \\
& K=-\left(t^{2}+x^{2}\right) \partial_{t}-2 r(t-r) \partial_{r}-2(t-r) x \partial_{x}
\end{aligned}
$$

In non-relativistic limit

$$
t \rightarrow t \quad r \rightarrow r \quad x \rightarrow \epsilon x
$$

we obtain the contracted Killing vectors

$$
\begin{array}{ccc}
P=\partial_{x} \quad B=(t-r) \partial_{x} & K_{x}=\left(t^{2}-2 t r\right) \partial_{x} & H=-\partial_{t} \\
D=-t \partial_{t}-r \partial_{r}-x \partial_{x} & K=-t^{2} \partial_{t}-2(t-r)\left(r \partial_{r}+x \partial_{x}\right)
\end{array}
$$

We can define an infinite extension of these vector fields in the bulk [3]

$$
\begin{aligned}
& M^{(n)}=\left(t^{n+1}-(n+1) r t^{n}\right) \partial_{x} \\
& L^{(n)}=-t^{n+1} \partial_{t}-(n+1)\left(t^{n}-n r t^{n-1}\right)\left(x \partial_{x}+r \partial_{r}\right)
\end{aligned}
$$

These vector fields obey the commutation relation (4) (without central charges). We can see that these vector fields at the boundary $r=0$ reduce to Killing vectors of contracted $C F T_{2}(3)$. The vector fields $M^{(n)}$ only 
act on the spatial coordinate $x$, so if we have a boundary condition at surface $x=0(z=\bar{z})$, these vector fields are removed from all Killing vectors in the bulk. Now we consider the action of the Virasoro generators $L^{(n)}$ (remanded Killing vectors) on $A d S_{3}$ metric (33) in non-relativistic limit. We introduce non-relativistic limit of $A d S_{3}$ metric which is given by $A d S_{2} \times R$ metric [3]

$$
d s^{2}=\frac{1}{r^{2}}\left(-2 d t d r+d t^{2}+d x^{2}\right) \rightarrow \frac{1}{r^{2}}\left(-2 d t d r+d t^{2}\right)
$$

The components of the metric in the $(t, r)$ directions survive and we receive to $A d S_{2}$ metric. The spatial direction $x$ is fiber over this $A d S_{2}$. Virasoro generators $L^{(n)}$ act non-trivially on all coordinate

$$
\begin{aligned}
& r \rightarrow r^{\prime}=r\left(1+a_{n}(n+1)\left(t^{n}-n r t^{n-1}\right)\right) \\
& t \rightarrow t^{\prime}=t\left(1+a_{n} t^{n}\right)
\end{aligned}
$$

where $a_{n}$ is infinitesimal parameter. From above equation we have

$$
\begin{aligned}
d r \rightarrow d r^{\prime} & =d r\left(1+a_{n}(n+1)\left(t^{n}-n r t^{n-1}\right)\right) \\
& +r a_{n} n(n+1) t^{n-2}((t-(n-1) r) d t-t d r) \\
d t \rightarrow d t^{\prime}= & d t\left(1+(n+1) a_{n} t^{n}\right)
\end{aligned}
$$

So in non-relativistic limit (35) we get

$$
d s^{2}=\frac{1}{r^{2}}\left(-2 d t d r+d t^{2}\right) \rightarrow \frac{1}{r^{2}}\left(-2 d t d r+d t^{2}+2 n\left(n^{2}-1\right) a_{n} r^{2} t^{n-2} d t^{2}\right)
$$

The $S L(2, R)$ subgroup which is generated by $L^{0}, L^{ \pm}$are exact isometries of non-relativistic version of boundary $A d S_{3}$. Near the boundary $r=0$ the diffeomorphisms of above metric has a fall-off like $r^{2}$, so other $L^{n}$ are asymptotic isometries of non-relativistic $A d S_{3}$. One copy of Virasoro algebra is asymptotic symmetry of non-relativistic $A d S_{3}$ metric with a boundary condition. Following [7, we calculate two-point function from gravity dual. Equation of motion for massive scalar field on the $A d S_{3}$ background (32) is given by

$$
\frac{1}{\sqrt{G}} \partial_{M}\left(\sqrt{G} G^{M N} \partial_{N} \phi(t, r, x)\right)-m^{2} \phi(t, r, x)=0
$$

In non-relativistic limit (35) we have

$$
\frac{1}{\sqrt{G}} \partial_{a}\left(\sqrt{G} g^{a b} \partial_{b} \phi\right)-m^{2} \phi=0 \quad \partial_{x}^{2} \phi=0
$$


The first equation can be obtained from the following action

$$
I=\int d t d r \sqrt{G} \frac{1}{2}\left(g^{a b} \partial_{a} \phi \partial_{b} \phi+m^{2} \phi^{2}\right)
$$

General solution of the equation of motion of the above action is

$$
\phi(t, r)=r e^{-i \omega t}\left(A I_{\alpha}(\omega r)+B K_{\alpha}(\omega r)\right)
$$

where $\alpha=\sqrt{m^{2}+1}$. From $A d S_{3} / C F T_{2}$ correspondence, we can find the bulk solution as

$$
\phi(t, r)=c \delta^{\Delta-2} \int d t^{\prime} \phi_{\delta}\left(t^{\prime}\right)\left(\frac{r}{r^{2}+\left|t-t^{\prime}\right|^{2}}\right)^{\Delta}
$$

where $\phi_{\delta}$ is a Dirichlet boundary value at $r=\delta$ and $\Delta=\alpha+\frac{1}{2}$. The above equation can be used to read two-point function of $G C A_{2}$

$$
<\phi\left(t_{1}\right) \phi\left(t_{2}\right)>\sim\left(t_{1}-t_{2}\right)^{-2 \Delta}
$$

which agrees with results (21) and (24).

\section{Conclusion}

Galilean conformal algebra (GCA) arises as a contraction of conformal algebra. We can use 2d GCA to constrain correlation functions. Correlation functions of Galilean conformal invariant fields in $2 \mathrm{~d}$ for space-time without boundary condition were found in [19]. We calculated two-point and threepoint functions in semi-infinite space with a boundary condition at surface $z=\bar{z}(x=0)$, by using some methods in quantum field theory (21) and from gravity dual (47). Our results (21) and (47) are agree with two-point function of BCFT in non-relativistic limit (24). We also introduce holographic dual of BCFT in non-relativistic limit (BGCA). AdS 3 with boundary condition and in non-relativistic limit has asymptotic isometries which are generated by one copy of non-relativistic version of Virasoro algebra .

\section{References}

[1] J. Lukierski, P. C. Stichel and W. J. Zakrzewski, Phys. Lett. A 357, 1 (2006); J. Gomis, J. Gomis and K. Kamimura, JHEP 0512, 024 (2005). 
[2] D. T. Son, Phys. Rev. D 78, 046003 (2008) arXiv:0804.3972 [hep-th]]; K. Balasubramanian and J. McGreevy, Phys. Rev. Lett. 101, 061601 (2008) [arXiv:0804.4053 [hep-th]]; W. D. Goldberger, arXiv:0806.2867 [hep-th]; J. L. B. Barbon and C. A. Fuertes, JHEP 0809, 030 (2008) arXiv:0806.3244 [hep-th]]; C. P. Herzog, M. Rangamani and S. F. Ross, arXiv:0807.1099 [hep-th]; A. Adams, K. Balasubramanian and J. McGreevy, arXiv:0807.1111 [hep-th].

[3] A. Bagchi and R. Gopakumar, JHEP 0907, 037, (2009).

[4] C. Duval and P. A. Horvathy, J. Phys. A42, 465206 (2009).

[5] D. T. Son, Phys. Rev. D 78, 046003 (2008).

[6] K. Balasubramanian and J. McGreevy, Phys. Rev. Lett. 101, 061601,(2008).

[7] M. Alishahiha, A. Davody and A. Vahedi, JHEP 0908, 022, (2009).

[8] A. Bagchi, A. Kundu, (2010), arXiv:1011.4999 [hep-th]].

[9] M. Henkel, J. Statist. Phys. 75 (1994).

[10] K. Binder, P. C. Hohenberg, Phys. Rev. B6, 3461 (1972); T. C. Lubensky, M. H. Rubin, Phys. Rev. Lett 31, 1469 (1973); T. C. Lubensky, M. H. Rubin, Phys. Rev. B ll, 4533 (1975); R. Lipowsky, Phys. Rev. Lett 49, 1575 (1982); H. W. Diehl, C. Domb, J. L. Lebowitz, Vol. 10, p. 75. London, Academic Press (1986).

[11] K. Binder, C. Domb, J. L. Lebowitz, Vol. 8, p. 1-144. London, Academic Press (1983)

[12] H. K. Janssen, B. Schaub and B. Schmittmann, Z. Phys. B73, 539 (1989).

[13] T. Takayanagi, Phys.Rev.Lett.107:101602,(2011).

[14] M. Fujita, T. Takayanagi and E. Tonni, "Aspect of AdS/BCFT," arXiv:1108.5152[hep-th]].

[15] J. L. Cardy, Nucl. Phys. B 240 (1984) 514; arXiv:hep-th/0411189;; D. M. McAvity and H. Osborn, Nucl. Phys. B 455, 522 (1995), arXiv:cond-mat/9505127]. 
[16] M. Alishahiha and R. Fareghbal, "Boundary CFT from Holography," arXiv:1108.5607 [hep-th]].

[17] M. R. Setare and V. Kamali, "Correlation functions of BCFT," arXiv:1109.3849 [hep-th]].

[18] A. Bagchi, R. Gopakumar, I. Mandal, A. Miwa, "GCA in 2d ", JHEP 1008:004,(2010), arXiv:0912.1090.

[19] A. Bagchi and I. Mandal, Phys. Lett. B 675, 393-397, (2009). A. Bagchi, (2010), arXiv:1012.3316]; M. R. Setare, V. Kamali, Int. J. Mod. Phys. A27, 1250044 (2012) [arXiv:1010.0329 [hep-th]].

[20] E. Kamke, Differentialgleichungen Lösungsmethoden und Lösungen, Vol. 2, 4th edition, Akademische Verlagsgesellschaft (Leipzig 1959). 